\title{
DISTRIBUIÇÃO DOS DISPOSITIVOS DE ACESSIBILIDADE NA REGIÃO CENTRAL DO MUNICÍPIO DE PRESIDENTE PRUDENTE-SP
}

\author{
Renan Furlan de Oliveira ${ }^{1}$, Mariana Dias Chaves ${ }^{2}$, Ana Paula da Silva Marques Ramos ${ }^{3}$ \\ 1,2 Universidade Estadual Paulista - UNESP, , Presidente Prudente, SP. ${ }^{3}$ Universidade do Oeste Paulista - UNOESTE, \\ Faculdade de Ciências, Letras e Educação - FACLEPP, Presidente Prudente, SP. E-mail: anaramos@unoeste.br, \\ mariana.unesp@hotmail.com
}

\section{RESUMO}

A Lei $n^{\circ} 10.098$ estabelece normas e critérios básicos para a promoção de acessibilidade às pessoas portadoras de deficiência física ou com mobilidade reduzida. De forma complementar, a NBR 9050 apresenta normas para a adoção de parâmetros de acessibilidade. Entretanto, alguns ambientes externos dos municípios, como vias, passeios, telefones públicos, nem sempre se encontram acessíveis. Uma vez que dispor de informações confiáveis e disponíveis sobre os dispositivos de acessibilidade pode proporcionar maior segurança ao usuário em termos de locomoção, o objetivo deste trabalho é representar espacialmente os dispositivos encontrados na região central da cidade de Presidente Prudente-SP e disponibilizar a representação ao usuário. Para isto, realizouse o levantamento de dados em campo, a construção de um banco de dados e a integração de diferentes fontes de informação. Os resultados mostram que embora os dispositivos estejam de acordo a lei e a norma, a distribuição espacial destes favorece regiões específicas. Dessa forma, a representação elaborada pode auxiliar em atividades de planejamento para os usuários, bem como para os órgãos de gestão municipais.

Palavras-chave: Deficiência física, acessibilidade, representação cartográfica e disponibilização de informação.

\section{DISTRIBUTION OF THE ACCESSIBILITY DEVICES ON THE DOWNTOWN OF PRESIDENTE PRUDENTE $-S P$}

\begin{abstract}
Law $n^{\circ} 10.098$ establishes standards and criteria for the promotion of accessibility for persons with disabilities or reduced mobility. As a complement, the NBR 9050 provides standards for the adoption of accessibility parameters. However, some external environments of cities, such as roads, sidewalks, public telephones, are not always accessible. Despite this, environments such as roads, sidewalks, public telephones, are not always accessible. .Once have reliable and available information on the accessibility devices can provide greater security to the user in terms of movement, the aim of this paper is represent the devices found in the downtown of Presidente Prudente-SP and provide representation to user. For this, it carried out the data collection in the field, building a database and the integration of different sources of information. The results show that although the devices are according to the law and the norm, the spatial distribution of these favors specific regions. Thus, the elaborate representation can assist in planning activities for users as well as for municipal governing bodies.
\end{abstract}

Keywords: Disability, accessibility, cartographic representation and providing information. 


\section{INTRODUÇÃO}

Conforme a Organização Mundial de Saúde (OMS), o portador de deficiência é aquele que apresenta um comprometimento físico, sensorial ou mental, que acarreta em limitação e o coloca em situação de desvantagem em relação à categoria das pessoas consideradas normais. No ano de 2010, uma pesquisa realizada pelo Instituto Brasileiro de Geografia e Estatística (IBGE), revelou que cerca de $23,9 \%$ dos brasileiros possui alguma deficiência e, comparada a pesquisas anteriores, este índice tem aumentado consideravelmente (IBGE, 2010). Dentre a população de deficientes enquadram-se os deficientes físicos, que enfrentam uma série de dificuldades para se locomover, por meio de muletas e (ou) cadeiras de rodas. Para indivíduos com esta limitação, o simples ato de sair de casa significa um desafio, sobretudo, porque a maioria das cidades brasileiras não dispõem de estrutura adequada para estes indivíduos (VASCONCELOS; PAGLIUCA, 2006).

Todas as pessoas têm direito ao acesso à educação, à saúde, à segurança, ao lazer e ao trabalho, o que contribui para a inserção social, desenvolvimento de uma vida saudável e de uma sociedade inclusiva (PAGLIUCA et al. 2007). Para o deficiente físico exercer seu direito e fortalecer sua participação como cidadão, é necessário que este tenha direito a acessibilidade em vias e edifícios de uso público e privado, bem como na utilização com segurança de equipamentos urbanos, meios de transportes e de comunicação (GODOY et al. 2000). No entanto, em vários segmentos da sociedade são notórias as inúmeras dificuldades enfrentadas pelos deficientes (CARVALHO, 2001), o que justifica a elaboração da Lei de Acessibilidade, número 10.098, de 19 de dezembro de 2000. Esta lei estabelece normas gerais e critérios básicos para a promoção da acessibilidade aos portadores de deficiência ou com mobilidade reduzida. Além disso, estabelece critérios para a adaptação de áreas de uso comum de edificações e fixa as condições exigíveis, padrões e medidas para proporcionar melhores condições e adequações para o acesso a edifícios e vias públicas. Dessa forma, todos os projetos dirigidos a deficientes devem seguir a Norma Brasileira (NBR) 9050, da Associação Brasileira de Normas Técnicas (ABNT), a qual padroniza as construções destinadas a este tipo de usuário.

Santos (2004) relata que a acessibilidade é um dos principais fatores que rege a interrelação entre a sociedade e o indivíduo portador de deficiência, influenciando em vários aspectos ligados a seus direitos enquanto cidadão. No entanto, verifica-se a falta de informações voltadas a esta problemática, principalmente na área de Usabilidade, no que diz respeito a relação do usuário com os produtos que utiliza e ambientes que frequenta, uma vez que estas informações seriam importantes para a concepção de dispositivos, mobiliários e ambientes de trabalho adequados (CARVALHO, 2001). 
A grande dificuldade por parte dos municípios em implantar e proporcionar à sociedade estes dispositivos chama a atenção. Além disso, alguns segmentos sociais que poderiam participar intensamente de atividades turísticas, culturais e de lazer oferecidas pelos municípios, deixam de participar, não apenas pela insuficiência de estrutura adequada às suas necessidades, mas também, principalmente, pela falta de informações sobre as possibilidades de acesso aos locais (DUARTE; COHEN, 2004). Neste sentido, com base nos capítulos II e IV da Lei n¹0.098 e na NBR 9050, o objetivo deste trabalho é realizar um estudo sobre os parâmetros técnicos de dispositivos de acessibilidade em circulações externas, com o intuito de representar espacialmente os dispositivos encontrados na região central da cidade de Presidente Prudente-SP e disponibilizar a representação ao usuário. Cabe ressaltar que para o desenvolvimento do presente trabalho foram utilizadas ferramentas da área de geotecnologias, como o Sistema de Informação Geográfica (SIG).

\section{METODOLOGIA}

Para construir a representação espacial dos dispositivos de acessibilidade da região central do município de Presidente Prudente e, posteriormente disponibilizá-la aos usuários, três grandes etapas foram realizadas: levantamento dos dispositivos de acessibilidade, criação e manipulação de um banco de dados geográficos e integração de fontes de informações e disponibilização do produto elaborado.

\section{Levantamento dos dispositivos de acessibilidade}

O levantamento dos dispositivos de acessibilidade abarcou as seguintes atividades: seleção da área de estudo, seleção dos dispositivos a serem representados, elaboração de formulários para coleta de informações em campo. A área de estudo consistiu na região central do município de Presidente Prudente, situado a oeste do estado de São Paulo. Esta região central é constituída por 25 quadras, localizada entre as quatro principais avenidas do município: Av. Washington Luiz, Av. Manoel Goulart, Av. Cel. José Soares Marcondes e Av. Brasil. Selecionou-se esta região devido ao fato da mesma ser composta por estabelecimentos de atendimento e prestação de serviço à população, o que reflete na circulação de um grande número de pessoas diariamente na região.

Após selecionar a área de estudo, foi realizado um estudo preliminar sobre os critérios e padrões apresentados na ABNT NBR 9050 para dar suporte a seleção dos tipos de dispositivos de acessibilidade à serem representados espacialmente. A partir deste estudo, decidiu-se considerar parâmetros de comunicação e acesso a circulação de pessoas com deficiência física ou mobilidade 
reduzida, abrangendo diferentes tipos de dispositivo de acessibilidade, como: telefones públicos, semáforos (ou focos de pedestres) e vagas para estacionamento de veículos.

Em seguida, foram elaborados formulários para coletar as informações em campo sobre cada tipo de dispositivo. As informações coletadas consistiram na quantidade de dispositivos e nos parâmetros determinados pela NBR 9050 no que diz respeito à acessibilidade a telefones públicos, semáforos e vagas para estacionamento de veículos. Em relação aos telefones públicos, verificouse a existência dos seguintes parâmetros: amplificador de sinal, sinalização sonora, sinalização do telefone e/ou sinalização de teclado. No que se refere aos semáforos, investigou-se a presença de dispositivos de acionamento de sinal e sinalização sonora. Para os semáforos com dispositivos de acionamento de sinal, foram coletadas informações sobre a altura destes dispositivos. Quanto às vagas para estacionamento de veículos que conduzam ou sejam conduzidos por pessoas com deficiência, verificou-se a existência dos seguintes parâmetros: sinalização horizontal e sinalização vertical. A existência das sinalizações, permitiu também a coleta do tipo de sinalização horizontal (paralela à calçada ou em $90^{\circ}$ ) e de sinalização vertical (símbolo de cadeirante ou símbolo E).

Os formulários permitiram a coleta de dados referentes ao número de identificação de cada dispositivo de acessibilidade e as informações referentes para cada tipo de dispositivo. 0 número de identificação foi utilizado para associar cada um dos dispositivos existentes à sua localização, uma vez que foi utilizado um croqui da região de estudo para auxiliar na coleta em campo. Após o levantamento dos dados, obteve-se um total de 10 telefones públicos, quatro semáforos e 19 vagas para estacionamento de veículos, distribuídos entre as 25 quadras da área de estudo. As informações coletadas referentes aos 33 dispositivos foram organizadas em forma de tabelas para serem armazenadas junto ao banco de dados geográficos, descrito adiante.

\section{Criação e manipulação do banco de dados geográficos}

A criação e a manipulação do banco de dados geográficos foram efetuadas no software ArcGIS, por meio de seus módulos ArcCatalog e ArcMap. Para isto, foram realizadas as seguintes atividades: criação do banco de dados, conversão dos dados de formato CAD (Computer Aided Design) para o formato SIG, edições geométricas dos dados, localização dos dispositivos de acessibilidade na base cartográfica correspondente à área de estudo e carga do banco de dados, por meio das tabelas de atributos referentes à cada tipo de dispositivo de acessibilidade.

A primeira atividade consistiu na criação de um banco de dados no ArcCatalog, que opera com dados em formato shapefile (.shp). Dessa forma, foi realizada a operação de conversão dos dados de formato CAD (.dwg) para o formato SIG (.shp), uma vez que a base cartográfica da região 
central do município de Presidente Prudente-SP, a qual foi disponibilizada para a realização deste trabalho, encontrava-se em formato CAD. A conversão foi realizada de tal modo que todas as informações contidas no arquivo de origem fossem preservadas. A partir disto, iniciou-se a edição geométrica dos dados no ArcMap.

A edição geométrica dos dados consistiu, basicamente, em excluir as informações consideradas desnecessárias para o desenvolvimento do trabalho. Dessa forma, mantiveram-se somente os dados correspondentes as quadras da região de estudo. Outra operação realizada foi a conversão de elementos lineares para polígonos, onde foi possível determinar alguns parâmetros para concretizar esta conversão de tal forma que não houvesse deslocamento dos vértices dos polígonos e não prejudicasse a área e a forma das quadras. Na sequência, foi possível localizar em cada uma das quadras, os diferentes dispositivos de acessibilidade considerados neste trabalho, os quais foram representados, inicialmente, por símbolos pontuais. A localização dos dispositivos de acessibilidade foi realizada alocando-se cada ponto de acordo com a localização anotada em campo no croqui da área de estudo. Após efetuar a representação espacial dos 33 dispositivos de acessibilidade, realizou-se a carga dos dados no banco de dados geográficos, isto é, a atribuição das informações e características a cada dispositivo, por meio das tabelas de atributos referentes aos dispositivos.

\section{Integração de fontes de informações e disponibilização do produto elaborado}

Com o intuito de integrar diferentes fontes de informações e disponibilizar a representação espacial, os arquivos em formatos (.shp), tais como as representações das quadras e dos tipos de dispositivos de acessibilidade, foram convertidos para o formato keyhole Markup Language (.kml), por meio do módulo ArcToolbox do ArcGIS. A conversão entre estes formatos permitiu com que os dados (.kml) fossem carregados e manipulados no Google Earth, o qual consiste em um aplicativo sem custo e de fácil acesso que permite a visualização de informações geográficas por usuários do mundo todo.

Após carregar os dados no aplicativo Google Earth, foram realizadas algumas edições das representações elaboradas no software ArcGIS. Uma dessas edições consistiu na substituição dos símbolos utilizados para a representação dos dispositivos de acessibilidade. No ambiente ArcMap, os dispositivos foram representados por pontos, enquanto que no Google Earth foi possível utilizar recursos do próprio aplicativo, atribuindo outros símbolos para as representações. Este recurso contribuiu com a utilização de símbolos mais intuitivos para o usuário, que ao selecionar qualquer 
tipo de símbolo na representação espacial, tem aceso às informações referentes ao dispositivo de acessibilidade em selecionado.

\section{RESULTADOS}

A integração de diferentes fontes de informações e a disponibilização de dados no Google Earth, foi realizada com o intuito de retratar a importância da disponibilização de informações por meio da internet, ao permitir que as representações elaboradas em softwares que não são de livre acesso, sejam utilizadas por usuários do mundo todo que necessitem dessas informações, por meio de um aplicativo sem custo, como relata Françoso et al. (2012). Sendo assim, a partir do resultado obtido (Figura 1), o usuário pode optar por qual tipo de informação deseja visualizar, possibilitando ainda, adicionar ao mapa o tipo de dispositivo de interesse para a visualização.

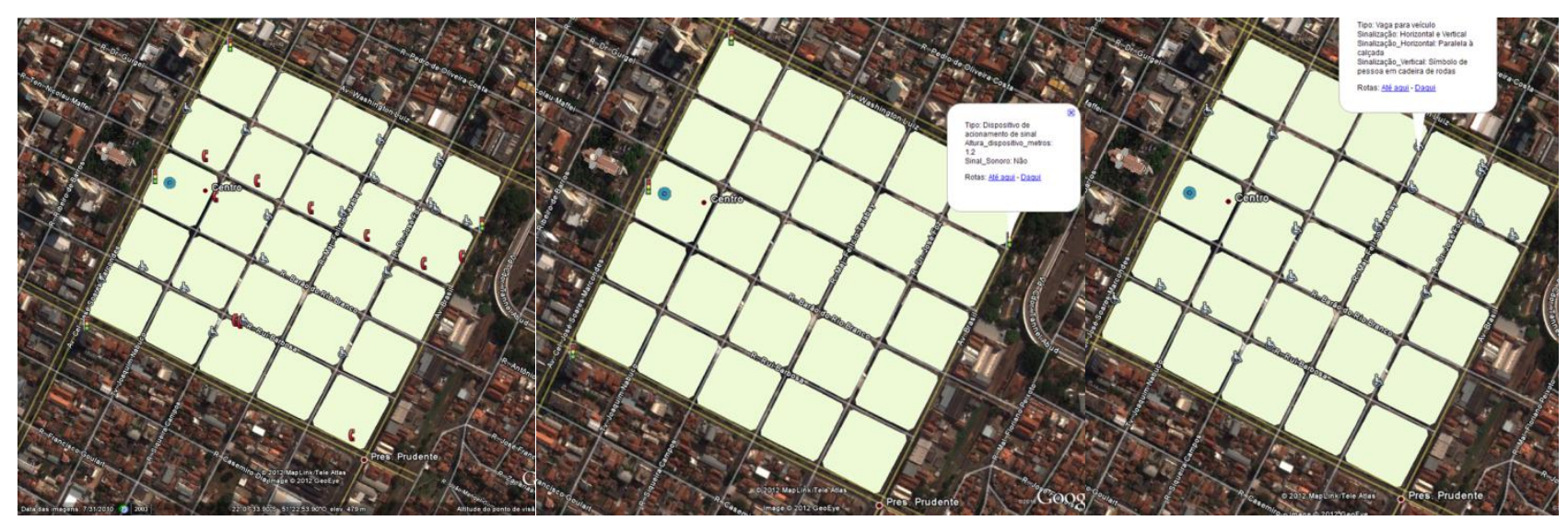

Figura 1. Disponibilização das representações no Google Earth.

\section{DISCUSSÃO}

A partir da representação espacial dos dispositivos correspondentes aos telefones públicos, foi possível identificar a ausência deste tipo de dispositivo com teclado e amplificador sonoro. Sendo assim, os telefones públicos encontrados na região de estudo atendiam apenas usuários cadeirantes.

Quanto à distribuição espacial deste tipo de dispositivo, pode-se verificar, a partir da Figura 1, que 50\% dos telefones estão presentes no "Calçadão" (Rua Tenente Nicolau Maffei), que passou por uma reforma a pouco tempo. Em relação aos semáforos adaptados aos portadores de deficiência, este tipo de dispositivo foi identificado apenas duas vezes e, deve ser tratado de modo especial, já que nas demais ruas e avenidas que contornam a região, trafega um grande fluxo de veículos diariamente e também apresentam semáforos, porém não se encontram adaptados. 
Quanto às vagas para estacionamento de veículos, este tipo de dispositivo foi encontrado com maior frequência (19) e encontra-se bem distribuído pela região. No entanto, apesar de estarem bem sinalizadas, o tipo de sinalização utilizado predominantemente foi o horizontal, o que pode trazer certo desconforto ao usuário, no momento de encontrá-lo.

\section{CONCLUSÃO}

Conclui-se que os dispositivos de acessibilidade em ambientes externos, encontrados na região central do município de Presidente Prudente-SP, seguem os padrões estabelecidos pela NBR 9050, fato importante e relevante aos usuários. As existências da Lei e da Norma contribuem para a fiscalização e a identificação dos dispositivos e norteiam os profissionais da área de geotecnologia a estruturar seus bancos de dados geográficos e definir correlações entre as informações, contribuindo com a forma de representação espacial dos dados.

O resultado obtido neste trabalho pode ser considerado um importante instrumento no que tange às questões de planejamento do espaço urbano. Isto porque permite investigar além da localização dos dispositivos de acessibilidade, os elementos que, indiretamente, contribuem para construção da real situação em termos de acessibilidade da região estudada, como por exemplo, a proximidade dos dispositivos quanto aos estabelecimentos comerciais e de prestação de serviços.

A representação espacial e a disponibilização das informações ao usuário, no Google Earth, contribui na identificação da distribuição espacial dos dispositivos de acessibilidade antes mesmo do usuário se deslocar de um lugar a outro. Assim, as informações disponibilizadas permitem que o usuário tenha um conhecimento prévio do local que pretende se locomover, evitando situações desconfortáveis e ainda, contribui para a economia de tempo e energia. Cabe ressaltar que a tecnologia utilizada neste trabalho, contribui também em atividades de planejamento e tomada de decisões por parte dos órgãos de gestão pública, como as prefeituras.

\section{REFERÊNCIAS}

Associação Brasileira de Normas Técnicas (ABNT). NBR 9050: Acessibilidade a edificações, mobiliário, espaços e equipamentos urbanos. 2004.

BRASIL. Lei n. 10.098, de 19 de dezembro de 2000. Estabelece normas gerais e critérios básicos para promoção de acessibilidade das pessoas portadoras de deficiência ou com mobilidade reduzida e dá outras providências [legislação na Internet]. Brasília. 2000.

CARVALHO, L. R. Ergonomia e o trabalho do portador de necessidade motora específica: o caso do cadeirante. Dissertação de Mestrado, Florianópolis, SC. 2001. 
DUARTE, C. R.; COHEN, R. Desenvolvimento de metodologia para confecção de um guia do Rio de Janeiro acessível. Anais do 2을 Seminário Internacional Visões Contemporânea. p. 1-13. Rio de Janeiro. 2004.

FRANÇOSO, M. T.; VALIN, M. M.; VICENTE, W. R.; COSTA, D. C.; TRABANCO, J. L. A. Disponibilização de informações sobre acessibilidade visão do promover a inclusão social. Revista MundoGEO. 2012.

GODOY, A.; NUNES, C. P.; REIS, D.A.; HATEM, D.S.; LORENTZ, L.N.; FERREIRA, M.J. Cartilha da inclusão dos direitos da pessoa com deficiência. Belo Horizonte: PUC/MG. 2000.

IBGE. Censo Populacional 2010. Instituto Brasileiro de Geografia e Estatística.

PAGLIUCA, L. M. F.; ARAGÃO, A. E. A.; ALMEIDA, P. C. Acessibilidade e deficiência física: identificação de barreiras arquitetônicas em áreas internar de hospitais de Sobral, Ceará. Revista da Escola de Enfermagem da USP, p. 581-588. 2007. http://dx.doi.org/10.1590/s0080$\underline{62342007000400007}$

SANTOS, L. K. S. Diretrizes de arquitetura e design para adaptação da habitação de interesse social ao cadeirante. Dissertação de Mestrado, Curitiba, PR. 2004.

VASCONCELOS, L. R.; PAGLIUCA, L. M. F. Mapeamento da acessibilidade do portador de limitação física a serviços básicos de saúde. EEAN - Escola de Enfermagem Anna Nery Revista de Enfermagem. p. $494-500.2006$. 